\title{
Allogeneic bone marrow transplant in pediatric patients in Kuwait
}

\begin{abstract}
To describe outcomes of human leukocyte antigen-matched sibling bone marrow transplantation (BMT) in 12patients: 9 with $\beta$-thalassemia major (TM) and 1 each with severe aplastic anemia, myelodysplastic syndrome and congenital dyserythropoietic anemia. The median age at transplantation was 9years and the median follow-up was 24 months. Among the TM patients, 6 were in Pesaro risk class II and 3 in class III. The median neutrophil recovery and platelet recovery were 19 and 25days, respectively. None of the patients had primary graft failure. At the last follow-up, 11 out of 12 patients were alive, free of disease and living normal quality of life. Most of the patients (75\%) had acute GVHD grade I-II, in the skin. None of the patients had grade IV regimen-related toxicity. Six patients developed Gram-negative while two had Gram-positive bacteremia, one had probable lung aspergillosis and four developed febrile neutropenia without identifiable microbiological growth during the aplastic phase. Cytomegalovirus reactivation occurred in five patients. We lost one patient who developed severe acute graft-versus-host disease (GVHD) affecting the skin, gut and liver, who later progressed to severe chronic GVHD and severe interstitial pneumonitis. In conclusion, allogenic BMT is feasible and safe in pediatric patients with hematological disorders in Kuwait.
\end{abstract}

Keywords: pediatric, bone marrow transplantation, Kuwait
Volume 2 Issue I - 2016

\author{
Al Sharida S,' Refaat S, ${ }^{2}$ Al Shemmari S,3 \\ 'Pediateric Hematology Unit, Mubarak hospital, Kuwait \\ ${ }^{2}$ Shaikha Badryia Stem Cell Transplant Center, Kuwait \\ ${ }^{3}$ Department of Medicine, Kuwait University Faculty of \\ Medicine, Kuwait
}

Correspondence: Sondus AI Sharidah. Department of Pediatrics, Faculty of Medicine, Kuwait University, State of Kuwait, P.O.BOX 24923-I3 I IO Safat, Telephone 965-6669I663, Email sundes72@yahoo.com

Received: December 07, 20I5 | Published: January 29, 2016
Abbreviations: BMT, bone marrow transplantation; TM, $\beta$-thalassemia major; GVHD, graft-versus-host disease; SCT, stem cell transplant; KCCC, kuwait cancer control center; SBH, sheika badryia hospital; SAA, severe aplastic anemia; MDS, myelodysplastic syndrome; CDA, congenital dyserythropoeitic anemia; HSCT, hematopoietic stem cell transplantation; HLA, human leukocyte antigen; $\mathrm{Cy}$, cyclophosphamide; $\mathrm{Bu}$, busulphan; $\mathrm{AUC}$, area under curve; $\mathrm{HCV}$, hepatitis $\mathrm{C}$ virus; $\mathrm{TNC}$, total nucleated cell; $\mathrm{MNC}$, mono nucleated cell; AST, aspartate amino transferase; ALT, alanine amino transferase; MTX, combined methotrexate;

\section{Introduction}

The stem cell transplant (SCT) center in Sheika Badryia Hospital (SBH) in Kuwait was established in 2000, initially with autologous SCT, offering stem cell rescue for consolidation high-dose chemotherapy for hematologic and non-hematologic malignancies. The hospital is part of the Kuwait Cancer Control Center (KCCC), which is a WHO-recognized comprehensive cancer center with stateof-the-art facilities. Both adult and pediatric patients are referred from all 7 governmental hospitals in the country. SCT service is offered to indigenous and expatriate patients free of charge. While the current population of Kuwait is nearly 4.8 million, the average number of pediatric patients referred for SCT is about ten per year. Transplant patients are nursed in a HEPA-filter unit equipped with, positivepressure laminar airflow rooms. The unit works in collaboration with the central blood bank service, which offers pre-transplant patient and donor evaluation regarding extended RBC phenotyping, antibody screening, and transfusion support.

\section{Patients and methods}

This case series is a single center experience of 12 consecutive pediatric patients (aged 3 to 17, with a median of 9 years), fine had beta thalassemia major (TM) and 1 each had severe aplastic anemia (SAA), myelodysplastic syndrome (MDS) and congenital dyserythropoietic anemia (CDA), who underwent hematopoietic stem cell transplantation (HSCT) from a human leukocyte antigen (HLA)identical sibling. The parents singed an informed consent.

\section{Procedural considerations}

All TM patients were risk classified based on Pesaro standard pretransplant liver size evaluation by clinical examination, liver fibrosis evaluation on liver biopsy, and adequacy of iron chelation.

Treatment regimen: The conditioning regimen of the TM patients consisted of Busulphan intravenous, and thiotepa was added for class 3 patients or patients 4years old or younger (Table 1). The SAA patient was conditioned with Fludarabine, Cyclophosphamide (Cy) and anti-thymocyte globulin and the MDS patient was conditioned with intravenous $\mathrm{Bu}$ and $\mathrm{Cy}$ and anti-thymocyte globulin. Class 3 TM patients received preparation chemotherapy before conditioning, consisted of azathioprine, hydoxyurea and fludarabine to augment peri-transplant immunosuppression to decrease risk of graft rejection, in addition to intravenous desferrioxamine and hyper transfusion to maintain hemoglobin concentration $13-15 \mathrm{gm} / \mathrm{L}$.

Table I Thalassemia patients

\begin{tabular}{ll}
\hline Pesaro risk class & No. of patients \\
\hline Class II & 6 \\
Class III & 3
\end{tabular}

Busulphan $(\mathrm{Bu})$ doses were based on actual patient body weight, and were adjusted according to busulphan area under curve (AUC) with drug monitoring in the Faculty of Pharmacy, Kuwait University, using high-performance liquid chromatography coupled with mass 
spectrometry. No prophylaxis was given against veno-occlusive disease. Cyclophosphamide was given with mesna uroprotection and hyper hydration.

The NCI common toxicity criteria version 2 was used to score the conditioning regimen toxicity. Oral mucositis, central nervous system, gastrointestinal, hepatic, pulmonary, cardiac, and renal toxicity were evaluated during the first 30days. The results of the infection surveillance were reported, and patients were observed for Jones criteria for VOD diagnosis.

\section{Definition of outcomes}

The day of neutrophil engraftment was defined as the first of 3 consecutive days with an absolute neutrophil count of 500 or higher Platelet engraftment was defined as the first of 7 consecutive days with platelet counts higher than 20,000 without transfusion. Primary graft failure or rejection was defined by persistent pancytopenia with no evidence of hematologic recovery of donor cells beyond 28 days after transplantation and secondary graft failure by a rapid decrease in neutrophil count after successful engraftment. Percentage of donor/ recipient DNA was evaluated on bone marrow samples at day 20 posttransplant, using PCR-based analysis of short tandem repeats.

Chimerism analysis was subsequently performed on day 60,90 , 180, and 365 after transplantation. Acute and chronic graft versus host disease were diagnosed and graded according to consensus criteria. Patients were given anti infective prophylaxis against bacterial, viral and fungal infections until neutrophil engraftment. Patients were monitored weekly for the Epstein-Barr virus, cytomegalovirus, adenovirus, and BK virus in the blood and/or urine using reverse transcription PCR starting before conditioning until at least 100days after transplantation. Serum galactomannan was monitored weekly for diagnostic-driven management. Transfusion support was given as patient-directed blood product transfusion support.

\section{Results}

Six of the TM patients were classified as class II and 3 as class III Pesaro risk categories. All the patients had normal pre-transplant assessment including cardiac echocardiography, pulmonary function, thyroid and parathyroid function tests. The infection surveillance tests was negative. All patients were herpes virus and cytomegalovirus immunoglobulin $G$ positive. There was inadequate iron chelation therapy in all 9TM patients. Pre-transplant serum ferritin, ranged from $1400-6000 \mathrm{mcg} / \mathrm{L}$; patients had intensive chelation therapy prior to transplant to decrease the ferritin to $<2000 \mathrm{mcg} / \mathrm{L}$. There was significant red blood cell transfusion exposure ( $>20 \mathrm{RBC}$ units) before BMT in all except the SAA patient. Only one patient had a pre-transplantation serological evidence of hepatitis $\mathrm{C}$ virus (HCV), however HCV-RNA by reverse transcription PCR was below detection limits. The cell dose: The total nucleated cell (TNC) dose infused was an average of $4.8 \times 108 / \mathrm{kg}$ (range $1.44-7.5 \times 108 / \mathrm{kg}$ ), mono nucleated cell (MNC) dose averaged $1.8 \times 108 / \mathrm{kg}$ (range $0.97-2.3 \times 108 / \mathrm{kg}$ ), and CD34+cell dose averaged 6.8x106/kg (range 3.5-14x106/kg) (Table 2).

Table 2 Cell dose for all patient

\begin{tabular}{ll}
\hline Cell dose & \\
\hline $\mathrm{NC}, 10^{8} / \mathrm{kg}$ & $4.8 \times 10^{8} / \mathrm{kg}$ \\
$\mathrm{CD} 34,10^{6} / \mathrm{kg}$ & $6.8 \times 10^{6} / \mathrm{kg}$ \\
\hline
\end{tabular}

\section{Engraftment data}

All 12patients had sustained engraftment. The median time to ANC $\geq 500 \times 109 / \mathrm{L}$ was 19 days (range, 13-22days), and median time to a platelet count $>20 \times 109 / \mathrm{L}$ was 25 days (range, 18-28 days). At 20days after transplantation, all patients had full donor chimerism (93\%-100\%) and 1 patient had $89 \%$ donor chimerism. At 60days after transplantation, all patients had $100 \%$ donor chimerism.

Regimen-related toxicities: No Busulphan related toxicity was observed. One patient developed moderate hepatic VOD, of moderate degree which resolved within 7days with supportive care. 10/12 patients had grade 2 hepatic toxicity with aspartate aminotransferase (AST) and alanine aminotransferase (ALT) elevations, stomatitis maximum grade 3, diarrhea, and cyclophosphamide related early hemorrhagic cystitis.

Six out of the 12patients developed neurological toxicity secondary to calcineurin inhibitors namely cyclosporine, in the form of hypertension (with encephalopathy and convulsions in 3patients). Two out of these 6patients developed further neurological toxicity to tacrolimus and were eventually shifted to mycophenolate mofetil.

Six patients developed Gram-negative, while 2 had Gram-positive bacteremia, 1 had probable lung aspergillosis and, 4 developed febrile neutropenia without identifiable microbiological grow during the neutropenic phase. Cytomegalovirus reactivation occurred in 5patients. Eight patients $(75 \%)$ had acute GVHD skin of grade I-II treated well with topical steroids. However, One TM class III patient had grade II chronic GVHD skin, but responded to systemic steroids within one week followed by gradual tapering. Another TM class III patient had severe acute stage III GVHD in the skin, gut and liver (stage III-IV) initially stabilized with maximum immunosuppression but later progressed to severe chronic GVHD of skin and liver. She also developed steroid-induced diabetes mellitus, and avascular bone necrosis at multiple sites (bilateral humeral heads, knees, and ankles), tacrolimus neurotoxicity, and interstitial pneumonia. Secondary graft failure occurred and marrow aplasia developed, and the patient died from respiratory failure on day 264-post transplantation.

Overall and disease-free survival: Total of 11patients are alive at last follow-up with a median follow up of 24 months, ranging between 11 months and 40months. ${ }^{1}$ They are all free of disease with normal quality of life.

\section{Discussion}

In this study, we have described the most recent transplantation experience in Kuwait in children with hematological disorders, who were treated with HLA-matched sibling BMT. ${ }^{1}$ It generates new back ground information about transplantation risk, which would assist in optimizing outcomes and selecting suitable candidates for transplantation. ${ }^{1}$ The results of TM patients must be interpreted in the light of recent advances in iron chelation therapy, and supportive therapy, which have reduced the morbidity and mortality risks in those who do not processed for transplantation. ${ }^{1-7}$ Our results confirm that HLA-matched sibling BMT for TM is a suitable therapeutic option to consider, as we also observed the tolerability of transplantation in children with reasonable good-risk features. ${ }^{1,8,9}$ These observations highlight the importance of carefully selecting good-risk patients for transplantation, as well as the modifications in the transplantation regimen that might improve the safety of the procedure. ${ }^{1,10-12}$ It appears that much of the toxicity of transplantation observed in this 
study might be related to impaired hepatic function and a propensity for developing interstitial pneumonitis, particularly in the class III TM patients. ${ }^{1}$ Studies in children with hematologic malignancies have demonstrated the utility of busulfan pharmacokinetics in predicting the risk of toxicity and have generally endorsed the adoption of targeted busulfan dosing. ${ }^{1,13,14}$ Moreover, a recent clinical study defined the important contribution by cyclophosphamide and its metabolites to the toxicity of busulfan, suggesting that the administration of busulfan before cyclophosphamide alters cyclophosphamide pharmacokinetics and therefore it might also exacerbate the risk of VOD in patients with underlying hepatic injury. ${ }^{1,15}$ To avoid these risks, investigative teams in Italy have reduced the dosing of cyclophosphamide in highrisk patients. ${ }^{1,3,12}$ Intravenous busulfan, in lieu of oral busulfan also appears to improve the safety profile of busulfan as does the practice of therapeutic drug monitoring. ${ }^{1,16}$ Several patient- and disease-related factors may influence outcome of transplantations for aplastic anemia, including recipient age, disease severity, numbers of pre transplantation transfusions, and donor-recipient sex-match., ${ }^{9}$ Major problems post transplantations are graft failure, GVHD, and infection. ${ }^{9}$ Graft failure can occur in $5 \%$ to $30 \%$ of patients. ${ }^{9}$ Combined methotrexate (MTX) and cyclosporine (CSA) is now the widely used regimen. ${ }^{9}$ Myelodysplastic syndrome (MDS) ${ }^{17}$ is a rare condition in childhood and often progresses to AML. Hematopoietic stem cell transplantation (HSCT) following myeloablative therapy is currently the only curative option in childhood MDS. The 3-year disease-free survival rate is around $50 \%$ in most series. ${ }^{18}$ HSCT has been successfully performed in other congenital anemias with dyserythropoiesis..$^{10,18}$ Using busulfan and cyclophosphamide for conditioning. ${ }^{1,10}$ Others have reported similar successful cases. ${ }^{10,18}$ More recently, Iolascon et al. ${ }^{19}$ reported successful stem cell transplantation in one case of severe, type II congenital dyserythropoietic anemia. ${ }^{10,18}$

\section{Conclusion}

In conclusion, the results of allogeneic SCT in the few patients in this report are gratifying and encouraging. The results also demonstrate the feasibility and relative safety of the procedure in Kuwait. Larger case series will be reported in the future.

\section{Acknowledgements}

None.

\section{Conflict of interest}

The author declares no conflict of interest.

\section{References}

1. Gaziev J, Sodani P, Lucarelli G. Hematopoietic stem cell transplantation in thalassemia. Bone Marrow Transplant. 2008;42(suppl 1):S41.

2. Sodani P, Gaziev J, Polchi P, et al. New approach for bone marrow transplantation in patients with class 3 thalassemia aged younger than 17 years. Blood. 2004;104(4):1201-1203.

3. Jones RJ, Lee KS, Beschorner WE, et al. eno-occlusive disease of the liver following bone marrow transplantation. Transplantation. 1987;44(6):778-783.

4. Przepiorka D, Weisdorf D, Martin P, et al. 1994 Consensus Conference on Acute GVHD. Bone Marrow Transplant. 1995;15(6):825-828.
5. Cappellini MD, Cohen A, Piga A, et al. A phase 3 study of deferasirox (ICL670), a once-daily oral iron chelator, in patients with betathalassemia. Blood. 2006;107(9):3455-3462.

6. Borgna-Pignatti C, Cappellini MD, De Stefano P, et al. Cardiac morbidity and mortality in deferoxamine- or deferiprone-treated patients with thalassemia major. Blood. 2006;107(9):3733-3737.

7. Maggio A, Vitrano A, Capra M, et al. Improving survival with deferiprone treatment in patients with thalassemia major: a prospective multicenter randomised clinical trial under the auspices of the Italian Society for Thalassemia and Hemoglobinopathies. Blood Cells Mol Dis. 2009;42(3):247-251.

8. Lucarelli G, Galimberti M, Polchi P, et al. Bone marrow transplantation in patients with thalassemia. $N$ Engl J Med. 1990;322(7):417-421.

9. Angelucci E, Baronciani D. Allogeneic stem cell transplantation for thalassemia major. Haematologica. 2008;93(12):1780-1784.

10. Hongeng S, Pakakasama S, Chuansumrit A, et al. Reduced intensity stem cell transplantation for treatment of class 3 Lucarelli severe thalassemia patients. Am J Hematol. 2007;82(12):1095-1098.

11. Bornhauser M, Storer B, Slattery JT, et al. Conditioning with fludarabine and targeted busulfan for transplantation of allogeneic hematopoietic stem cells. Blood. 2003;102(3):820-826.

12. Young NS, Barrett AJ. The treatment of severe acquired aplastic anemia. Blood. 1995;85:3367-3377.

13. Chiesa R, Cappelli B, Crocchiolo R, et al. Unpredictability of intravenous busulfan pharmacokinetics in children undergoing hematopoietic stem cell transplantation for advanced beta thalassemia: limited toxicity with a dose-adjustment policy. Biol Blood Marrow Transplant. 2010;16(5):622628.

14. Chandy M, Balasubramanian P, Ramachandran SV, et al. Randomized trial of two different conditioning regimens for bone marrow transplantation in thalassemia: the role of busulfan pharmacokinetics in determining outcome. Bone Marrow Transplant. 2005;36(10):839-845.

15. McCune JS, Batchelder A, Deeg HJ, et al. (Cyclophosphamide following targeted oral busulfan as conditioning for hematopoietic cell transplantation: pharmacokinetics, liver toxicity, and mortality. Biol Blood Marrow Transplant. 2007;13(7):853-862.

16. Gaziev J, Nguyen L, Puozzo C, et al. Novel pharmacokinetic behavior of intravenous busulfan in children with thalassemia undergoing hematopoietic stem cell transplantation: a prospective evaluation of pharmacokinetic and pharmacodynamic profile with therapeutic drug monitoring. Blood. 2010;115(22):459714604.

17. McCann SR, Bacigalupo A, Gluckman E, et al. Graft rejection and second bone marrow transplants for acquired aplastic anaemia: A report from the Aplastic anaemia working party of the european bone marrow transplant group. Bone Marrow Transplant. 1994;13(3):233-237.

18. Strahm B, N öllke P, Zecca M, et al. EWOG-MDS study group. Hematopoietic stem cell transplantation for advanced myelodysplastic syndrome in children: results of the EWOG-MDS 98 study. Leukemia. 2011;25(3):455-462.

19. Iolascon A, Sabato V, De Mattia D, et al. Bone marrow transplantation in a case of severe, type II congenital dyserythropoietic anaemia (CDA II). Bone Marrow Transplant. 2001;27(2):213-221. 